\title{
IkasLagun Posta. Una iniciativa de refuerzo académico en los recursos residenciales de Gipuzkoa tras el COVID-19
}

\author{
Joana Miguelena Torrado'; Joxe Garmendia Larrañaga²; Luis María Naya Garmendia³; Pauli Dávila \\ Balsera $^{4}$
}

En esta colaboración presentamos una iniciativa, IkasLagun Posta, que ha servido como "cierre" de otro programa, IkasLagun (en euskera acompañante de estudios), que ha estado en vigor los dos últimos cursos académicos. IkasLagun tiene como objetivo ofrecer refuerzo académico a niñas y niños que están bajo la medida de protección del acogimiento residencial en Gipuzkoa (España). Esta iniciativa viene a colación del COVID-19, que ha imposibilitado que este refuerzo académico haya podido seguir dándose, debido a las consecuencias de la declaración de la pandemia y el estado de alarma. El programa IkasLagun es un programa de voluntariado donde estudiantes de la Universidad del País Vasco/Euskal Herriko Unibertsitatea (UPV/EHU) acuden semanalmente a ofrecer refuerzo académico a niñas y niños atendidos en acogimiento residencial. El cierre obligatorio de las universidades y de centros escolares y el posterior confinamiento han ocasionado el fin del programa en este curso académico sin un "cierre" adecuado entre el alumnado voluntario y las niñas y niños.

\section{La educación como un reto}

La educación y el nivel formativo, como se ha demostrado en multitud de estudios, son factores que favorecen o dificultan las situaciones de inclusión o exclusión social (Subirats, 2004). No obstante, en el caso de las niñas, niños y adolescentes bajo alguna medida de protección, se convierte en un factor protector clave (Mallon, 2007), principalmente, porque al cumplir la mayoría de edad, se produce su obligada salida del sistema de protección y el comienzo de una acelerada transición a una vida adulta llena de complejidades, dificultades y desventajas (Stein, 2005; Goyette, 2010; Jariot, Sala-Roca, Arnau, 2015; Melendro, 2016; Dixon, 2016). Asimismo, el ámbito educativo de las niñas, niños y adolescentes en acogimiento residencial es un reto escasamente asumido (González, 2018), pero no solamente la educación, sino también la salud mental, el acceso al mundo laboral, las relaciones familiares, el apoyo social y otros riesgos como la maternidad/paternidad temprana, problemas con la justicia, demanda de ayudas sociales o el horizonte nada deseable de convertirse en indigentes (López et al, 2013).

Este reto cobra importancia y demanda una iniciativa permanente en cada investigación en la que se analiza el ámbito educativo de las niñas, niños y adolescentes en acogimiento residencial o de jóvenes egresados del sistema de protección. De hecho, la mayoría de ellas ponen de manifiesto que este colectivo experimenta numerosos desafíos educativos como un peor nivel formativo (Tilbury, 2010), ya que no suelen estar en el nivel que les corresponde por edad, y acostumbran a contar con un número mayor de repeticiones o incluso, presentan un mayor porcentaje de absentismo escolar que la población normativa (Martin, Jackson, 2002; Dell'Aglio, Hutz, 2004; Siqueira, Dell, 2010; Montserrat, Casas, Baena, 2015). Por lo tanto, no es necesario insistir en que se trata de un grupo de personas vulnerables y donde alcanzar un mejor nivel educativo puede ayudarles en una carrera más exitosa.

\footnotetext{
Universidad del País Vasco/Euskal Herriko Unibertsitatea. E-mail: joana.miguelena@ehu.eus

2 Universidad del País Vasco/Euskal Herriko Unibertsitatea. E-mail: joxe.garmendia@ehu.eus

Universidad del País Vasco/Euskal Herriko Unibertsitatea.

E-mail: luisma.naya@ehu.eus

4 Universidad del País Vasco/Euskal Herriko Unibertsitatea.

E-mail: pauli.davila@ehu.eus
}

Soc. Infanc. 4, 2020: 185-288 


\section{Arrakasta: un programa para garantizar el acceso a la universidad}

El origen del programa IkasLagun tiene su génesis en el Programa para el éxito académico Arrakasta (éxito en euskera) que, a su vez, surgió de una tesis doctoral que se desarrolló en el seno del Grupo de Estudios Históricos y Comparados en Educación-Garaian (Garaian). En dicha tesis doctoral se analizaban los derechos de las niñas, niños y adolescentes bajo la medida de protección de Gipuzkoa, poniendo una especial atención en los derechos de participación y de educación. En ella se pudieron identificar las dificultades que los jóvenes extutelados tienen para poder cursar estudios universitarios y mantenerse en la universidad hasta su finalización. Ante esta situación, el grupo Garaian consiguió concitar a diversos agentes: la UPV/EHU, la Diputación Foral de Gipuzkoa (DFG) y la Fundación $\mathrm{SM}$, poniendo en marcha un programa pionero que crea y/o refuerza las condiciones existentes para que este colectivo en desigualdad de condiciones pueda cursar y finalizar sus estudios universitarios, ofreciendo apoyo logístico, académico, económico y habitacional, entre otros (Miguelena, Dávila, Naya, 2018).

Como han evidenciado distintos estudios, la universidad no es un itinerario habitual entre las personas que egresan del sistema de protección (Martin, Jackson, 2002; Cashmore, Paxman, Townsend, 2007; Montserrat, Casas, Malo, Bertrán, 2011; Jackson, Cameron, 2012; Harvey, McNamara et al. 2015; Miguelena, Dávila, Naya, 2018; Miguelena, 2019), siendo mucho más comunes itinerarios formativos de corta duración y profesionalizantes que les ayuden a una rápida inserción laboral al alcanzar su mayoría de edad, momento en el que están obligados a dejar el sistema de protección y comenzar una vida independiente: la temida edad de los 18 años.

La puesta en marcha del programa Arrakasta, proporcionaba medios para que un pequeño porcentaje de jóvenes extutelados que llegaban a la universidad no vieran truncado su futuro por cuestiones exclusivamente económicas. Una vez garantizado que, después del acceso a la universidad, podían seguir una carrera en igualdad de condiciones que el resto de estudiantes universitarios, surgió un nuevo reto: ¿qué podíamos hacer para que llegase a la universidad o cursar otro itinerario formativo más largo un número mayor de niños, niñas y adolescentes que están en el sistema de protección sin que esto fuera un factor condicionante de no hacerlo? Se propuso abordar un programa en el que, a través de una especie de aprendizaje-servicio el alumnado universitario, apoyáramos académicamente a quienes estaban en los niveles de enseñanza no universitaria.

\section{IkasLagun: apoyo académico para la igualdad de oportunidades}

Garaian, junto con la Sección de acogimiento residencial de la DFG, comenzó a esbozar un programa que ayudase a paliar la desigualdad de oportunidades educativas de este colectivo, ofreciendo refuerzo académico individualizado a las niñas y niños bajo tutela (dos horas por semana) en los propios recursos de protección. Los beneficios de este programa parecían evidentes en tres elementos clave del proceso de enseñanza-aprendizaje. Por una parte, en relación a los beneficios de las niñas y niños, les ayudaría a mejorar su rendimiento académico y a ampliar su red de apoyo. Asimismo, esta relación educativa tiene un valor añadido, al favorecer un acompañamiento personalizado entre el niño o niña y el Ikaslagunkide (el compañero del programa IkasLagun) y mejorar sus competencias lingüísticas en euskera.

Por otra parte, el alumnado de la UPV/EHU adquiere competencias personales, académicas y sociales; descubre nuevas realidades sociales que les permitirán desempeñar mejor su profesión en el futuro; contribuye a una garantía de la igualdad de oportunidades educativas y, además, recibe un reconocimiento académico de 3 créditos.

Finalmente, el tercer beneficiario de este programa de un modo directo son los equipos educativos de los recursos residenciales, al mitigar su carga laboral en lo referido al apoyo académico de las niñas y niños bajo su guarda.

El programa se puso en marcha por primera vez en el curso académico 2018/2019. Ese curso 28 niñas y niños fueron atendidos en 5 recursos residenciales por 32 voluntarios y voluntarias y, fueron supervisados, con una labor de seguimiento, por miembros de Garaian y de la DFG, siempre con el apoyo, no solamente económico, de la Fundación SM. Para la puesta en marcha del programa, se hizo una presentación pública del mismo, en la que el alumnado interesado recibía información sobre los diferentes aspectos del programa. Una vez alcanzado un compromiso, el alumnado debía entregar el certificado de delitos de carácter sexual, acudir a unas sesiones de formación y a la acogida en el recurso residencial previa a conocer a la niña o niño asignado. La asignación siguió tanto los criterios de las características del refuerzo académico (idioma, curso académico, asignaturas, etc.) como de la agenda de ambos. Tras la evaluación del primer año, se destacaron como beneficios los siguientes: la personalización de la relación educativa, la mejora del rendimiento académico general, y especialmente la mejora de la competencia lingüística del idioma euskera, ayudando a los niños y niñas a tener una actitud mucho más positiva y disminuir el rechazo hacia el euskera. Otro factor clave fue el del cambio de expectativas en torno al futuro académico del alumnado. Por parte de los Ikaslankide, se apreció un acercamiento a una realidad desconocida, una empatía con los niños y niñas, profundizar en aprendizajes docentes de tipo individualizado e incluso unas expectativas laborales.

Tras analizar la evaluación del programa, sus puntos fuertes y los de mejora, todas las partes consideramos que debíamos seguir con el programa durante el siguiente curso académico. Por ello, en el mes de septiembre de 2019 hicimos una convocatoria para llevar a cabo el reclutamiento de alumnado, para ello contamos con la colaboración de diversos centros de la UPV/EHU que se encargaron de enviar la convocatoria a los correos electrónicos corporativos del alumnado. Más de 150 alumnas y alumnos de distintas disciplinas mostraron su interés por participar en IkasLa- 
gun. La posibilidad de contar con este incremento de alumnado voluntario fue decisiva para ampliar el programa y proponer a la DFG la ampliación del programa, ofreciendo refuerzo académico a todas aquellas niñas y niños de hasta 12 años atendidos en recursos de protección que lo necesitaran.

Finalmente, el programa IkasLagun 2019-20 comenzó su andadura con 64 niñas y niños atendidos en 12 recursos de protección y 64 voluntarias y voluntarios y otros 54 suplentes. IkasLagun, en un curso académico, ha crecido exponencialmente, de tal manera que la UPV/EHU y la DFG se han implicado para redactar un convenio de colaboración. La presencia del grupo Garaian está garantizada asumiendo labores de promoción, seguimiento y evaluación del programa. El COVID-19 ha supuesto el cierre del sistema educativo y de las universidades el día 13 de marzo. Como en el caso de la mayoría de las prácticas académicas, y de otros proyectos y programas, el cese inmediato de la actividad no permitió una despedida ni "cierre" presencial del programa. No obstante, la declaración del estado de alarma ha evidenciado la actitud positiva con la que las niñas, niños y adolescentes bajo tutela están afrontando el confinamiento que, al contrario que en la mayoría de los hogares que están confinados con sus familias, estas y estos no han podido ver a las suyas, ya que las visitas familiares han tenido que ser suspendidas.

\section{IkasLagun Posta: una respuesta al COVID-19}

En esta nueva situación, en la que las niñas y niños de hasta 12 años bajo la tutela no tienen móvil, el alumnado de la UPV/EHU, si quería comunicarse y saber de las niñas y niños, debían llamar al recurso residencial o ponerse en contacto con el grupo Garaian. No se trataba únicamente de seguir con el programa académico, sino de continuar una relación personal truncada.

Dada la cercanía y el trato directo con el grupo Garaian, el alumnado de la UPV/EHU comenzó, desde el inicio del confinamiento, a preguntar por la situación de las niñas y niños. Para responder a esta necesidad que, por otra parte, también sabíamos que era vivida de la misma manera por los niños y niñas, pusimos en marcha una nueva iniciativa: IkasLagun Posta (acompañante de estudio por correo). En ella, pedimos a todo el alumnado de la UPV/EHU que se pusiera en contacto mediante correo electrónico con el niño o niña al que le ofrecía refuerzo hasta la semana anterior. Por su parte, desde los recursos asistenciales confirmamos que la niña o el niño iba a escribir otra carta a su IkasLagun. En el caso de quienes sabían mecanografiar, la escribieron adjuntándola en un email; quienes no sabían, la escribieron en un papel y los equipos educativos las escanearon y se las remitieron a los IkasLagun.

Actualmente, esta relación no ha cesado en la mayoría de los casos, haciendo uso, incluso de videollamadas, emails, WhatsApp a través de los teléfonos de los equipos educativos, etc. Es decir, se ha utilizado toda la tecnología que hemos tenido a nuestra disposición para que el programa no terminara de una manera tan abrupta. Esta reacción espontánea muestra el nivel de implicación de todas las personas involucradas en el mismo. No podemos olvidar que ha existido un alto número de IkasLagunkide que ha repetido programa o muestra su interés en continuar.

\section{Hacia un futuro cercano}

La incertidumbre de cómo será el programa en el curso académico 2020/2021 es el escenario en el que nos encontramos a corto y medio plazo. No obstante, este escenario no nos impide saber que el programa IkasLagun seguirá caminando de un modo presencial, semi-presencial u online, contribuyendo a paliar la desigualdad de oportunidades educativas, apostando por el derecho a la educación como eje de nuestro cometido. El espíritu solidario del alumnado de la UPV/EHU, el trabajo del grupo Garaian y la colaboración institucional de la UPV/EHU, la DFG y la Fundación SM van a ser las piezas de un nuevo futuro para los niños, niñas y adolescentes en situación de protección y construir un horizonte de mayor igualdad de oportunidades en el ámbito educativo.

\section{Referencias bibliográficas}

Cashmore, J., Paxman, M., Townsend, M. (2007). The educational outcomes of young people 4-5 years after leaving care: An Australian perspective. Adoption \& Fostering, 31(1), 50-61.

Dell'Aglio, D., Hutz, C. S. (2004). Depressão e desempenho escolar em crianças e adolescentes institucionalizados. Psicologia: Reflexão e Crítica, 17(3), 341-350.

González, P. (2018). El Acogimiento Residencial Infantil en Tenerife: perfiles atendidos, programas de intervención y evaluación de resultados (Tesis doctoral). Universidad de la Laguna, La Laguna (en línea). https://riull.ull.es/xmlui/handle/915/9446

Goyette, M. (2010). El tránsito a la vida adulta de los jóvenes atendidos desde servicios sociales. Pedagogía Social. Revista Interuniversitaria, 17, 43-56.

Harvey, A., Andrewartha, L., McNamara, P. (2015). A forgotten cohort? Including people from out-of-home care in Australian higher education policy. Australian Journal of Education, 59(2), 182-195.

Jackson, S., Cameron, C. (2012). Final report of the YIPEE project. Young people from a public care background: Pathways to further and higher education in five European countries. London: Thomas Coram Research Unit. 
Jariot, M., Sala-Roca, J., Arnau, L. (2015). Jóvenes tutelados y transición a la vida independiente: indicadores de éxito. Revista Española de Orientación y Psicopedagogía (REOP), 26(2), 90-103.

López, M., Santos, I., Bravo, A., del Valle, J. (2013). El proceso de transición a la vida adulta de jóvenes acogidos en el sistema de protección infantil. Anales de psicología, 29(1), 187-196.

Mallon, J. (2007). Returning to education after care: protective factors in the development of resilience. Adoption \& Fostering, 31(1), 106-117.

Martin, P. Y., Jackson, S. (2002). Educational success for children in public care: Advice from a group of high achievers. Child \& Family Social Work, 7(2), 121-130.

Miguelena, J. (2019). Los derechos de las niñas, niños y adolescentes atendidos en recursos residenciales de acogida de programa básico en Gipuzkoa: representaciones sociales y propuestas de mejora (Tesis doctoral). Universidad del País Vasco/ Euskal Herriko Unibertsitatea. Donostia-San Sebastián.

Miguelena, J., Dávila, P., Naya, L. M. (2018). Éxito universitario en el País Vasco: el programa Arrakasta. En XVI Congreso Nacional Educación Comparada Tenerife Educación e Inclusión: Aportes y perspectivas de la Educación Comparada para la Equidad (pp. 433-442). La Laguna: Universidad de La Laguna.

Montserrat, C., Casas, F., Baena, M. (2015). L'educació dels infants i adolescents en el sistema de protecció. Un problema o una oportunitat? Girona: Documenta Universitaria.

Montserrat, C., Casas, F., Malo, S., Bertrán, I. (2011). Los itinerarios educativos de los jóvenes ex-tutelados. Madrid: Ministerio de Sanidad, Política Social e Igualdad.

Siqueira, A. C., Dell, D. D. (2010). Crianças e adolescentes institucionalizados: desempenho escolar, satisfação de vida e rede de apoio social. Psicologia: Teoria e Pesquisa, 26(3), 407-415.

Stein, M. (2005). Young people aging out of care: The poverty of theory. Children and Youth Services Review, 28(4), 422-434

Tilbury, C. (2010). Educational status of children and young people in care. Children Australia, 35, 7-13. 